\title{
Lysophosphatidic Acid Level and the Incidence of Silent Brain Infarction in Patients with Nonvalvular Atrial Fibrillation
}

\section{Zhen-Guang Li, Zhan-Cai Yu, Yong-Peng Yu *, Wei-Ping Ju, Dao-Zhen Wang, Xia Zhan, Xi-Juan Wu and Li Zhou}

Department of Neurology, Wendeng Center Hospital of Weihai, the Affiliated Hospital of Weifang Medical College, Weihai, Shandong 264400, China; E-Mails: lzg6598@ 163169.net (Z.-G.L.); wdchywk@163.com (Z.-C.Y.); jwpz67@126.com (W.-P.J.); zhenwd17@126.com (D.-Z.W.); wdzhx686@sina.com (X.Z.); wxj7979@163.com (X.-J.W.); drlizhou2009@126.com (L.Z.)

* Author to whom correspondence should be addressed; E-Mail: yypeng6688@126.com; Tel.: +86-0631-8474031; Fax: +86-0631-8476036.

Received: 12 August 2010; in revised form: 25 September 2010 / Accepted: 12 October 2010 / Published: 19 October 2010

\begin{abstract}
Lysophosphatidic acid (LPA), which is proposed to play an important role in normal physiological situations such as wound healing, vascular tone, vascular integrity and reproduction, may be involved in the etiology of some diseases such as atherosclerosis, cancer, obesity or myocardial infarction. Abnormal findings, including silent brain infarction (SBI), are frequently observed by magnetic resonance imaging (MRI) in patients with nonvalvular atrial fibrillation (NVAF). However, whether there is a relationship between LPA level and the prevalence of SBI has not been extensively studied. In the present study, the association between them was investigated. 235 patients with NVAF, 116 cases of SBI without NVAF and 120 cases of healthy volunteers (control group), who did not receive any antithrombotic therapy, were enrolled in this study. Plasma LPA levels in the NVAF with SBI group were significantly higher than that in the control group $(p<0.01)$, NVAF without SBI group $(p<0.01)$ and SBI without NVAF group $(p<0.01)$. The LPA levels are lower in the control group than in the NVAF without SBI and SBI without NVAF groups $(p<0.01)$, however, the latter two groups did not significantly differ from each other for LPA levels $(p>0.05)$ There were significant differences in the positive rate of platelet activation between each of the groups $(p<0.01)$. The positive rate of platelet activation was significantly higher in the NVAF with SBI group. We suggest that LPA might be a novel marker for estimation of the status of platelet activation and the risk
\end{abstract}


factor for SBI onset in NVAF patients. We expected that plasma LPA levels could predict the occurrence of SBI in NVAF patients.

Keywords: atrial fibrillation; lysophospholipids; ischemic stroke; platelet activation

\section{Introduction}

Atrial fibrillation (AF) is the most common sustained arrhythmia and its incidence increases with age $[1,2]$. The incidence of stroke increased by 5-7 times in patients with AF in contrast to that with no AF $[3,4]$. Therefore, it seems particularly important to explore the mechanism of emboli formation in non-valvular atrial fibrillation (NVAF) patients. Previous studies mainly concentrated on the coagulation and fibrinolytic functions. However, few studies have addressed the platelet activation state in patients with NVAF in vivo since there are few molecular markers which can effectively reflect the platelet activation in vivo [5]. In recent years, studies have found that lysophosphatidic acid (LPA), as a multifunctional "phospholipid messenger", is mainly produced by activated platelets and may be an ideal molecular marker [6,7] reflecting platelet activation state in vivo. Platelets contribute to the production of LPA [8], which has been proposed to be a primary lipid in atherosclerotic plaque that is responsible for platelet activation [9]. LPA is also abundant in human atherosclerotic plaques, where it is thought to be derived, at least in part, from mild oxidation of low density lipoproteins [10]. Platelet depletion or treatment with anti-platelet agents reduces circulating LPA levels in animals [11,12]. Hence, anti-thrombotic therapy targeting LPA production or receptor-mediated signaling may be a novel strategy to prevent thrombus formation. Silent brain infarction (SBI), which increases vascular cognitive impairment and stroke risk, has a high incidence in elderly people [13,14]. The occurrence of stroke in patients with NVAF is likely preceded by subclinical cerebrovascular disease. Many potentially abnormal findings, including the presence of SBI, which is detectable with brain magnetic resonance imaging (MRI), have been recognized in patients without clinically apparent previous cerebrovascular accidents [15-17]. Despite their high prevalence and clinical significance, the pathogenesis of silent ischemic lesions still remains elusive, especially in patients with NVAF [18]. There is consensus that it is high in elderly subjects and patients with risk factors for stroke such as hypertension, age and AF [16,19]. As with coronary artery disease, multiple observational or cross-sectional studies have shown a relationship between homocysteine (Hcy) and stroke [20-24]. In addition, a Japanese study found a relationship between Hcy and silent areas of infarction by MRI, that is to say, patients with a silent stroke also had high Hcy levels [14]. Other studies reported the incidence of SBI to be 2.5 to 4.5 times higher in hyperhomocysteinemia [14], which is an independent risk factor for vascular diseases such as cerebrovascular and cardiovascular diseases [25,26]. Furthermore, SBI has been considered as a risk factor for brain hemorrhage and symptomatic infarction [19]. Futoshi et al. [27] indicated that there was a significant association between plasma homocysteine level and the prevalence of SBI in hemodialysis patients with chronic renal failure. However, the significance of increased plasma LPA with SBI in NVAF patients has not been adequately investigated. We hypothesized that increased levels of LPA are associated with SBI in 
NVAF patients, which might provide basis for clinical antithrombotic therapy targeting LPA production and its clinical application.

\section{Results and Discussion}

\subsection{Results}

\subsubsection{Baseline Characteristics of the Study Groups}

The baseline characteristics of each gGroup are shown in Table 1. Clinical and laboratory data, including age, sex, the occurrence of hypertension, diabetes mellitus and hyperlipidemia, and smoking status were not different in the four groups.

Table 1. Baseline characteristics of each group.

\begin{tabular}{lllccc}
\hline Variable & $\begin{array}{l}\text { NVAF + SBI }(+) \\
(\mathbf{n = 7 4 )}\end{array}$ & $\begin{array}{l}\text { NVAF + SBI }(-) \\
(\mathbf{n = 1 6 1})\end{array}$ & $\begin{array}{l}\text { SBI + NVAF (-) } \\
(\mathbf{n = 1 1 6})\end{array}$ & $\begin{array}{l}\text { Control } \\
(\mathbf{n = 1 2 0})\end{array}$ & $\boldsymbol{p}$ \\
\hline Age, years (mean + SD) & $64.6 \pm 6.5$ & $65.6 \pm 7.9$ & $62.5 \pm 7.6$ & $66.9 \pm 5.8$ & $\mathrm{~ns}$ \\
Male & $39(52.7 \%)$ & $83(51.6 \%)$ & $65(56.0 \%)$ & $64(53.3 \%)$ & $\mathrm{ns}$ \\
Hypertension & $38(51.4 \%)$ & $78(48.4 \%)$ & $59(50.9 \%)$ & $51(42.5 \%)$ & $\mathrm{ns}$ \\
Diabetes mellitus & $17(23.0 \%)$ & $36(22.4 \%)$ & $25(21.6 \%)$ & $22(18.3 \%)$ & $\mathrm{ns}$ \\
Dyslipidemia & $20(27.0 \%)$ & $40(24.8 \%)$ & $28(24.1 \%)$ & $26(21.7 \%)$ & $\mathrm{ns}$ \\
Smoking & $13(17.6 \%)$ & $27(16.7 \%)$ & $20(17.2 \%)$ & $18(15.0 \%)$ & $\mathrm{ns}$ \\
Platelet count $\left(\times 10^{9}\right)$ & $197.1 \pm 38.7$ & $180.3 \pm 33.6$ & $203.2 \pm 40.5$ & $190.4 \pm 38.8$ & $\mathrm{~ns}$ \\
\hline
\end{tabular}

NVAF with SBI: NVAF + SBI (+); NVAF without SBI: NVAF + SBI (-); SBI without NVAF: $\mathrm{SBI}+\mathrm{NVAF}(-)$.

\subsubsection{Comparison of Plasma LPA Levels between Different Groups}

Among 235 cases of NVAF, 74 cases with SBI, which were found in accordance with the diagnostic criteria mentioned above, accounted for 31.5\%. Plasma LPA levels in the NVAF with SBI group were significantly higher than that in the control group $(p<0.01)$, NVAF without SBI group $(p<0.01)$ and SBI without NVAF group $(p<0.01)$. The LPA levels are lower in the control group than in the NVAF without SBI and SBI without NVAF groups $(p<0.01)$. However the latter two groups were not significantly different from each other for LPA levels $(p>0.05)$ (Figure 1).

\subsubsection{Incidence of NVAF with SBI}

There were 59 cases of NVAF with SBI out of 157 cases of NVAF in patients older than 60 years of age, which accounted for $37.6 \%$. In contrast, there were 15 cases of NVAF with SBI in 78 cases of NVAF patients younger than 60 years of age, which accounted for $19.2 \%$. For the control group, this percentage was $10.8 \%$. However, adjusting for age, the difference in the incidences of SBI in patients with NVAF older than 60 years of age compared with control groups remained statistically significant $(p<0.01)$. 
Figure 1. Comparison of LPA concentration ( $\mu \mathrm{mol} / \mathrm{L})$ between the different study groups: (A) NVAF with SBI; (B) NVAF without SBI; (C) SBI without NVAF; (D) Control. Data are presented as means \pm SEM. Columns relevant to each group bearing different superscripts $(\mathrm{a}, \mathrm{b}$ or $\mathrm{c})$ differ significantly $(p<0.01)$.

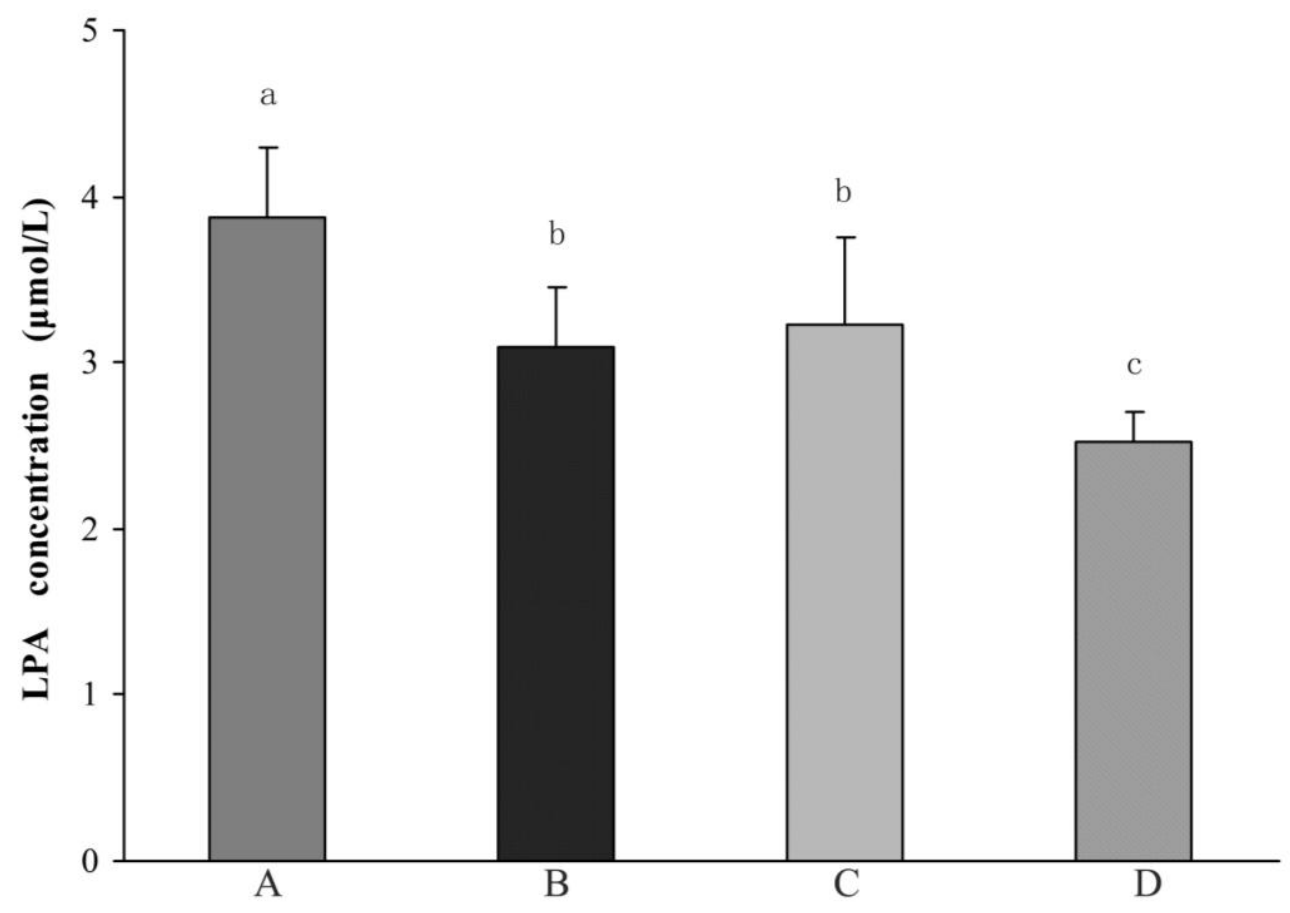

\subsubsection{Comparison in the Positive Rate of Platelet Activation}

The percentage of platelet activation classified into mild, moderate and severe degree in the NVAF with SBI group was $6.8 \%, 8.1 \%$ and $85.1 \%$ respectively. However these percentages were $46.0 \%$, $34.8 \%$ and $21.2 \%$, respectively, in the NVAF without SBI group. In parallel, the data was $22.4 \%$, $12.1 \%$ and $65.5 \%$ in the SBI without NVAF group and $79.2 \%, 12.5 \%, 8.3 \%$ in controls. There was a significant difference in the positive rate of platelet activation in each group $(p<0.01)$.

\subsection{Discussion}

The atrium loses its effective systolic function and the blood clots in theleft atrium at AF. Due to endothelial dysfunction, platelet activation, coagulation and fibrinolytic activity being abnormal, the body is in a state of hypercoagulability or prethrombotic and the left atrium, particularly in the left atrial appendage, easily form mural thrombus. Once the thrombus is detached, it causes thromboembolic complications, especially ischemic stroke [14]. According to previous study, though platelet activation was not directly involved in thrombosis in AF, it could play an important role in blood hypercoagulability [28]. The data in the present study indicated that plasma LPA levels were significantly increased in NVAF patients with SBI. It revealed that platelet activation existed in these patients and that this activation might contribute to further LPA generation, which would activate more platelets to form emboli or emboli rain in turns. The emboli lead to SBI. Thus LPA can be used as a possible molecular marker for estimating thrombosis or prethrombotic status in patients with NVAF. 
A Japanese community-based study reported that the incidence of SBI in the population of 66-year-old elderly was $24.8 \%$ [29], while the incidence of SBI in the population of 65-year-old elderly was $28 \%$ in a cardiovascular health study [30]. Our study found that the incidence of SBI in NVAF patients of 60 -years-old was $37.6 \%$, significantly higher than that in the age-matched control group $(10.8 \%)$. However, the prevalence of SBI in patients with NVAF in our study is higher than that in the common population [29]. It should be noted that this prevalence is related to the particular study subjects rather than true prevalence, because the present study has a possible selection bias inherent in this type of hospital-based study. The prevalence of SBI will be affected by the sensitivity of the examination technique and the composition of the study samples with regard to age, sex, race and vascular risk factors. In additional, some other serum markers such as homocysteine (Hcy) or brain natriuretic peptide (BNP), known to correlate with an increased incidence of SBI, might be involved in the alteration of LPA levels. Hyperhomocysteinemia also enhances platelet adhesion to endothelial cells, promotes growth of vascular smooth muscles cells, and is associated with higher levels of prothrombotic factors such as $\beta$-thromboglobulin, tissue plasminogen activator and factor VII coagulant (FVIIc) [27]. Although this issue was discussed controversially, previous study indicated that elevated plasma concentrations of BNP were associated with the presence of AF [31] while the associations between these serum markers and LPA levels had not been fully understood. The additional further research on the role that the interaction between these serum markers such as LPA, Hcy and BNP played in the prevalence of the SBI was desirable. Furthermore, AF was one of the most important risk factors for SBI. So the frequency of SBI in the present study was relatively higher than that in previous reports mentioned above. Moreover, previous studies reported that an irregular heart rate blunted nitric oxide (NO) synthesis and increased p-selectin expression on platelets, ultimately increasing the risk for SBI $[32,33]$. Irregular shear stress upon the vascular endothelium in patients with AF also may cause sclerotic changes which contribute to the attenuation of NO synthesis [34,35]. This hypothesis is further supported by several reports that the prevalence of SBI lesions is associated with atherosclerosis related factors such as aging, diabetes mellitus and hypertension [36-39]. If the NO attenuation hypothesis is true, the higher prevalence of SBI in the NVAF patients may be explained better.

One of limitations of our study was that the study design was not randomized. Secondly, an extension of the actual data by the determination of some additional already established markers for platelet activation such as granulocyte-colony stimulating factor (G-CSF), interleukin-6 and thrombopoietin (TPO), etc., might strengthen the result of this study. It was a pity that these markers were not determined in this study. Thirdly, certain diseases may influence activated LPA release, including inflammatory processes, hypercholesterolemia or diabetes mellitus. In additional, LPA was measured after SBI onset in this study. Whether high LPA can be a risk factor for SBI needs a prospective study. Future studies, therefore, need to include not only prospective or longitudinal LPA comparative analyses but also investigations that will determine whether plasma LPA levels are influenced by other medical conditions or biomarkers which affecting the production, secretion and circulation of LPA. Longitudinal studies are also required to assess how well LPA levels correlate with SBI and thus might be used as a marker for monitoring treatment, progression and recurrence. 


\section{Materials and Methods}

\subsection{Study Subjects}

Subjects enrolled in the study were informed and gave consent. The protocol of the study was approved by the local ethics committees. Patients were selected from 894 patients with NVAF who had been screened in outpatient clinics in four teaching hospitals. Of the 894 patients, 235 cases with NVAF and 116 cases of SBI without NVAF, who did not receive any antithrombotic therapy, were recruited as candidates for the present study. 120 cases of healthy volunteers older than 60 -years of age (control group) were enrolled in this study. The controls were selected by the same experienced clinician and were subjected to neurological examination, and were neurologically normal adults in regard to sinus rhythm, recruited during routine health check-ups at the neurology outpatient clinic. All subjects enrolled in this study underwent echocardiography and ultrasonography of the carotid arteries. The candidate criterion used to select 235 cases with NVAF is as following: (1) Continuous or intermittent NVAF in patients was diagnosed by clinical symptoms, electrocardiogram, cardiac ultrasound and so on; (2) Age varied from 45 to 85. Exclusion criteria: Patients with NVAF, who had any of the following illnesses, conditions or requirement: (1) Rheumatic or congenital valvular heart disease; (2) After heart valve replacement; (3) Congestive heart failure and severe heart diseases. The diagnosis of congestive heart failure and severe heart diseases was confirmed by a complete medical evaluation, medical history and physical examination as well as various tests including echocardiogram and a chest X-ray to detect abnormal function of the left ventricle and/or heart valves and the size and shape of the heart; (4) Coagulation abnormalities. Coagulation abnormalities was defined as an INR spontaneously $>1.6$ and/or an APTT $>60 \mathrm{~s}$ and/or a platelet count $<150 \times 10^{9} / \mathrm{L}$ and/or a fibrinogen less than $1.0 \mathrm{~g} / \mathrm{L}$; (5) Intracranial or systemic infection; (6) Obvious organs dysfunction (such as liver, kidney, lung). The cut off values about organs dysfunction were shown in Table 2; (7) With previous history of stroke or transient ischemic attack (TIA); (8) Receiving antithrombotic or warfarin therapy; (9) Hypertension with carotid atherosclerosis or hemal stricture revealed by carotid color ultrasonic examination; (10) Female in the menstruation period. 116 cases of SBI without NVAF were selected from patients who were hospitalized with dizziness, headache, cervical spondylosis, lumbar spinal stenosis, intracranial infection, acute carbon monoxide poisoning, facial paralysis and so on. A normal neurological examination with no focal deficit at the time of entry was required. 120 healthy controls that had no history of cerebral or cardiac vascular diseases and no symptoms mentioned above within one month were matched to patients by age and gender.

Information about clinical characteristics in each group such as arterial hypertension $(\mathrm{AH})$, diabetes mellitus (DM), dyslipidemia and smoking habit were recorded. Cranial MRI was performed in all participants at entry to the study. Because this study involved multiple institutions, the MRI instruments differed, and a magnetic field strength of $1.0 \mathrm{~T}$ or more was used at each institution. Almost all patients underwent MRI examination, except for two patients in the SBI group who had metal in their bodies, and were analyzed on the basis of cerebral computed tomography without MRI. All subjects were examined by the same investigator who was blinded to clinical characteristics. The diagnosis of SBI was made with reference to the criteria established by Matsui et al. [14,40]: (1) SBI was defined as an area ( $\geq 3 \mathrm{~mm}$ in diameter) of focal hyperintensity on T2-weighted images or FLAIR 
images with corresponding low signal intensity on T1-weighted images and low density lesions on CT; (2) Lack of evidences for the nervous system symptoms or signs which can be explained by lesions observed on MRI or CT; (3) Without a history of clinical stroke; (4) Patients with micro-bleeding or suspected bleeding followed by infarction were excluded by imaging.

Table 2. The cut-off values for organ dysfunction.

\begin{tabular}{ll}
\hline Variables & Cut off Values \\
\hline Respiratory, $\mathrm{PaO}_{2} / \mathrm{FiO}_{2}(\mathrm{mmHg})$ & $\leq 300$ \\
Coagulation, platelets $\times 10^{3} / \mu \mathrm{mol}$ & $\leq 150$ \\
Liver, bilirubin $(\mathrm{mg} / \mathrm{dL})^{\mathrm{a}}$ & $\geq 2.0$ \\
Cardiovascular, hypotension & Mean arterial pressure $<70 \mathrm{mmHg}$ \\
Renal creatinine $(\mathrm{mg} / \mathrm{dL})^{\mathrm{b}}$ or & $>2.0$ or \\
Urine output $(\mathrm{mL} / \mathrm{d})$ & $<500 \mathrm{~mL}$ \\
Glomerular filtration rate $(\mathrm{GFR})$ & $\leq 70 \mathrm{~mL} / \mathrm{min}$ \\
\hline
\end{tabular}

${ }^{\mathrm{a}}$ To convert bilirubin from $\mathrm{mg} / \mathrm{dL}$ to $\mu \mathrm{mol} / \mathrm{L}$, multiply by $17.1 ;{ }^{\mathrm{b}}$ To convert creatinine from $\mathrm{mg} / \mathrm{dL}$ to $\mu \mathrm{mol} / \mathrm{L}$, multiply by 88.4 .

\subsection{Sample Collection and LPA Analysis}

Patients were told to stay away from fatty food and alcohol for several days before fasting blood samples were obtained in the morning. We did not have blood drawn from women if they were in their menstruation period. Four milliliters of venous blood was drawn from each participant into commercially available anticoagulant tubes (Two-fish Comp.). Plasma was either processed immediately or stored at $-70{ }^{\circ} \mathrm{C}$ before lipid extraction. Lipid extraction was performed at $0{ }^{\circ} \mathrm{C}$ to $4{ }^{\circ} \mathrm{C}$ to minimize damage to ester bonds, using a slight modification of published methods [41]. Whole blood was centrifuged at $3500 \mathrm{~g}$ for 10 minutes. The supernatant was transferred to a microcentrifuge tube and centrifuged at $8000 \mathrm{~g}$ for 10 minutes. Then $1 \mathrm{~mL}$ suspension (platelet-poor plasma) was used for the remaining procedure. Lipids were extracted with $4 \mathrm{~mL}$ of 1-butanol and $2 \mathrm{~mL}$ of water-saturated 1-butanol. The resulting organic extracts were pooled and dried in vacuo. Each sample was resuspended in $0.3 \mathrm{~mL}$ of chloroform/methanol/water/28\% $\mathrm{NH}_{4} \mathrm{OH}$ (250:100:15:0.3, v/v) and was immediately filtered by an Econosphere $3 \mu \mathrm{m}, 50 \times 4.6 \mathrm{~mm}$ silica column (Alltech Associates, Deerfield, IL). Compounds were eluted with a mobile phase of chloroform/methanol/water/28\% $\mathrm{NH} 4 \mathrm{OH}(250: 100: 15: 0.3 \mathrm{v} / \mathrm{v})$ at $0.5 \mathrm{~mL} / \mathrm{min}$. The source was maintained at $250{ }^{\circ} \mathrm{C}$ with a drying gas flow of $10 \mathrm{~L} / \mathrm{h}$. The concentration of LPA was quantified by measuring its inorganic phosphorus component using colorimetric assays.

\subsection{Estimation of Platelet Activation Degree}

Plasma LPA was considered to be a more sensitive and meaningful biomarker of platelet activation and LPA level could indirectly reflect the degree of platelet activation in vivo. The cut-off values for LPA levels were classified according to the degree of platelet activation in this study as follows: If the LPA concentration $\leq 3.0 \mu \mathrm{mol} / \mathrm{L}$, platelet function was considered as being in a state of normal or mild activation; If LPA concentration $>3.0 \mu \mathrm{mol} / \mathrm{L}$, but $\leq 3.5 \mu \mathrm{mol} / \mathrm{L}$, the platelet function was in moderate 
activation state; LPA concentration $>3.5 \mu \mathrm{mol} / \mathrm{L}$, the platelet function was in a highly activated state or the formation of emboli. The data about the risk classification in respect to the LPA plasma levels is based on our previous study [42]. However, these cut-off values may be applicable only to this study population and will have to be reevaluated following larger studies.

\subsection{Statistical Analysis}

Comparisons of the baseline characteristics and observations in the present study were performed by ANOVA and univariate analysis with Student's t-test or Mann-Whitney's test for continuous variables and the Fisher exact test for noncontinuous variables. Chi-square test was used to analyze the incidence of SBI between the NVAF group and the control group. All statistical analyses were performed with SPSS software package for Windows version 11.5. Data was expressed as mean \pm SEM and statistical significance was set at $p<0.05$.

\section{Conclusions}

Our data indicate that the plasma LPA levels significantly increased in NVAF or NVAF patients with SBI, which was accompanied by the high incidence of SBI. Based on the results presented here, we suggest that LPA might be a novel marker for estimation of the status of platelet activation and the risk factor for SBI onset in NVAF patients. We expect that plasma LPA levels could predict the occurrence of SBI in NVAF patients. There may be a relationship between plasma LPA levels and the prevalence of SBI in NVAF patients. If the plasma LPA levels were not an independent predictor for SBI, its predicted significance may be limited and this data should be interpreted carefully because the present study was not designed to test the predictabilities of plasma LPA levels. However, our results warrant further study. This was also the first study to examine the association between plasma LPA levels and the occurrence of SBI in NVAF patients and it might be an important subject for randomized trials and prospective research for some years to come.

\section{Acknowledgements}

This study was supported by grants from the Chinese Science and Technology Ministry (G2000056905).

\section{References}

1. Kopecky, S.L.; Gersh, B.J.; McGoon, M.D.; Whisnant, J.P.; Holmes, D.R., Jr.; Ilstrup, D.M.; Frye, R.L. The natural history of lone atrial fibrillation: a population-based study over three decades. N. Engl. J. Med. 1987, 7, 669-674.

2. Ma, C.S.; Du, X.; Jiang, C.X. Atrial fibrillation in China: a brief review. Chin. Med. J. (Engl.) 2009, 122, 2803-2806.

3. Hart, R.G.; Pearce, L.A. Current status of stroke risk stratification in patients with atrial fibrillation. Stroke 2009, 40, 2607-2610.

4. Ahmad, O.; Ahmad, K.E.; Dear, K.B.; Harvey, I.; Hughes, A.; Lueck, C.J. Atrial fibrillation and anticoagulation in a stroke unit population. Intern. Med. J. 2009, 39, 752-756. 
5. Kamath, S.; Blann, A.D.; Lip, G.Y. Platelets and atrial fibrillation. Eur. Heart J. 2001, 22, 2233-2242.

6. Pamuklar, Z.; Federico, L.; Liu, S.; Umezu-Goto, M.; Dong, A.; Panchatcharam, M.; Fulerson, Z.; Berdyshev, E.; Natarajan, V.; Fang, X.; van Meeteren, L.A.; Moolenaar, W.H.; Mills, G.B.; Morris, A.J.; Smyth, S.S. Autotaxin/Lysopholipase D and Lysophosphatidic acid regulate murine hemostasis and thrombosis. J. Biol. Chem. 2009, 284, 7385-7394.

7. Smyth, S.S.; Cheng, H.Y.; Miriyala, S.; Panchatcharam, M.; Morris, A.J. Roles of lysophosphatidic acid in cardiovascular physiology and disease. Biochim. Biophys. Acta 2008, 1781, 563-570.

8. Eichholtz, T.; Jalink, K.; Fahrenfort, I.; Moolenaar, W.H. The bioactive phospholipid lysophosphatidic acid is released from activated platelets. Biochem. J. 1993, 291, 677-680.

9. Rother, E.; Brandl, R.; Baker, D.L.; Goyal, P.; Gebhard, H.; Tigyi, G.; Siess, W. Subtype-selective antagonists of lysophosphatidic acid receptors inhibit platelet activation triggered by the lipid core of atherosclerotic plaques. Circulation 2003, 108, 741-747.

10. Siess, W.; Zangl, K.J.; Essler, M.; Bauer, M.; Brandl, R.; Corrinth, C.; Bittman, R.; Tigyi, G.; Aepfelbacher, M. Lysophosphatidic acid mediates the rapid activation of platelets and endothelial cells by mildly oxidized low density lipoprotein and accumulates in human atherosclerotic lesions. Proc. Natl. Acad. Sci. USA 1999, 96, 6931-6936.

11. Aoki, J.; Taira, A.; Takanezawa, Y.; Kishi, Y.; Hama, K.; Kishimoto, T.; Mizuno, K.; Saku, K.; Taguchi, R.; Arai, H. Serum lysophosphatidic acid is produced through diverse phospholipase pathways. J. Biol. Chem. 2002, 277, 48737-48744.

12. Boucharaba, A.; Serre, C.M.; Gres, S.; Saulnier-Blache, J.S.; Bordet, J.C.; Guglielmi, J.; Clezardin, P.; Peyruchaud, O. Platelet-derived lysophosphatidic acid supports the progression of osteolytic bone metastases in breast cancer. J. Clin. Invest. 2004, 114, 1714-1725.

13. Vermeer, S.E.; Longstreth, W.T., Jr.; Koudstaal, P.J. Silent brain infarcts: a systematic review. Lancet Neurol. 2007, 6, 611-619.

14. Matsui, T.; Arai, H.; Yuzuriha, T.; Yao, H.; Miura, M.; Hashimoto, S.; Higuchi, S.; Matsushita, S.; Morikawa, M.; Kato, A.; Sasaki, H. Elevated plasma homocysteine levels and risk of silent brain infarction in elderly people. Stroke 2001, 32, 1116-1119.

15. Uehara, T.; Tabuchi, M.; Mori, E. Risk factors for silent cerebral infarsts in subcortical white matter and basal ganglia. Stroke 1999, 30, 378-382.

16. Lee, S.C.; Park, S.J.; Ki, H.K.; Gwon, H.C.; Chung, C.S.; Byun, H.S.; Shin, K.J.; Shin, M.H.; Lee, W.R. Prevalence and risk factors of silent cerebral infarction in apparently normal adults. Hypertension 2000, 36, 73-77.

17. Adachi, T.; Kobayashi, S.; Yamaguchi, S.; Okada, K. MRI findings of small subcortical 'lacunar-like' infarction resulting from large vessel disease. J. Neurol. 2000, 247, 280-285.

18. Narumiya, T.; Sakamaki, T.; Sato, Y.; Kanmatsuse, K. Relationship between left atrial appendage function and left atrial thrombus in patients with nonvalvular chronic atrial fibrillation and atrial flutter. Circ. J. 2003, 67, 68-72.

19. Kobayasi, S.; Okada, K.; Koide, H.; Bokua, H.; Yamaguti, S. Subcortical silent brain infarction as a risk factor for clinical stroke. Stroke 1997, 28, 1932-1939. 
20. Coull, B.M.; Malinow, M.R.; Beamer, N.; Sexton, G.; Nordt, F.; de Garmo, P. Elevated plasma homocyst(e)ine concentration as a possible independent risk factor for stroke. Stroke 1990, 21, 572-576.

21. Giles, W.H.; Croft, J.B.; Greenlund, K.J.; Ford, E.S.; Kittner, S.J. Total homocysteine concentration and the likelihood of nonfatal stroke: Results from the Third National Health and Nutrition Examination Survey, 1988-1994. Stroke 1998, 29, 2473-2477.

22. Boushey, C.J.; Beresford, S.A.; Omenn, G.S.; Motulsky, A.G. A quantitative assessment of plasma homocysteine as a risk factor for vascular disease. Probable benefits of increasing folic acid intakes. JAMA 1995, 274, 1049-1057.

23. Moller, J.; Nielsen, G.M.; Tvedegaard, K.C.; Andersen, N.T.; Jorgensen, P.E. A meta analysis of cerebrovascular disease and hyperhomocystinemia. Scand. J. Clin. Lab. Invest. 2000, 60, 491-499.

24. Hankey, G.J.; Eikelboom, J.W. Homocysteine and stroke. Curr. Opin. Neurol. 2001, 14, 95-102.

25. Homocysteine Studies Collaboration. Homocysteine and risk of ischemic heart disease and stroke: a meta-analysis. JAMA 2002, 288, 2015-2022.

26. Wald, D.S.; Law, M.; Morris, J.K. Homocysteine and cardiovascular disease: evidence on causality from a meta-analysis. BMJ 2002, 325, 1202-1206.

27. Anan, F.; Takahashi, N.; Shimomura, T.; Imagawa, M.; Yufu, K.; Nawata, T.; Nakagawa, M.; Yonemochi, H.; Eshima, N.; Saikawa, T.; Yoshimatsu, H. Hyperhomocysteinemia is a significant risk factor for silent cerebral infarction in patients with chronic renal failure undergoing hemodialysis. Metabolism 2006, 55, 656-661.

28. Kamath, S.; Blann, A.D.; Chin, B.S.; Lanza, F.; Aleil, B.; Cazenave, J.P.; Lip, G.Y. A study of platelet activation in atrial fibrillation and the effects of antithrombotic therapy. Eur. Heart J. 2002, 23, 1788-1795.

29. Shinkawa, A.; Ueda, K.; Kiyohara, Y.; Kato, I.; Sueishi, K.; Tsuneyoshi, M.; Fujishima, M. Silent cerebral infarction in a community-based autopsy series in Japan. The Hisayama Study. Stroke 1995, 26, 380-385.

30. Price, T.R.; Manolio, T.A.; Kronmal, R.A.; Kittner, S.J.; Yue, N.C.; Robbins, J.; Anton-Culver, H.; Oleary, D.H. Silent brain infarction on magnetic resonance imaging and neurological abnormalities in community dwelling older adults: the Cardiovascular Health Study. CHS collaborative research group. Stroke 1997, 28, 1158-1164.

31. Gartner, W.; Zierhut, B.; Mineva, I.; Sodeck, G.; Leutmezer, F.; Domanovits, H.; Prayer, D.; Wolf, F.; Base, W.; Weissel, M.; Wagner, L. Brain natriuretic peptide correlates with the extent of atrial fibrillation-associated silent brain lesions. Clin. Biochem. 2008, 41, 1434-1439.

32. Minamino, T.; Kitakaze, M.; Sato, H.; Asanuma, H.; Funaya, H.; Koretsune, Y.; Hori, M. Plasma levels of nitrite/nitrate and platelet cGMP levels are decreased in patients with atrial fibrillation. Arterioscler. Thromb. Vasc. Biol. 1997, 17, 3191-3195.

33. Minamino, T.; Kitakaze, M.; Sanada, S.; Asanuama, H.; Kurotobi, T.; Koretsune, Y.; Fukunami, M.; Kuzuya, T.; Hoki, N.; Hori, M. Increased expression of P-selectin on platelets is a risk factor for silent cerebral infarction in patients with atrial fibrillation: role of nitric oxide. Circulation 1998, 98, 1721-1727. 
34. Noris, M.; Morigi, M.; Donadelli, R.; Aiello, S.; Foppolo, M.; Todeschini, M.; Orisio, S.; Remuzzi, G.; Remuzzi, A. Nitric oxide synthesis by cultured endothelial cell is modulated by flow condition. Circ. Res. 1995, 76, 536-543.

35. Topper, J.; Cai, J.; Falb, D.; Gimborne, M. Identification of vascular endothelial genes differentially responsive to fluid mechanical stimuli: cyclooxygenase-2, manganese superoxide dismutase, and endothelial cell nitric oxide synthase are selectively up-regulated by steady laminar shear stress. Proc. Natl. Acad. Sci. USA 1996, 93, 10417-10422.

36. Uehara, T.; Tabuchi, M.; Mori, E. Risk factors for silent cerebral infarsts in subcortical white matter and basal ganglia. Stroke 1999, 30, 378-382.

37. Lindgren, A.; Roijer, A.; Rudling, O.; Norrving, B.; Larsson, E.M.; Eskilsson, J.; Wallin, L.; Olsson, B.; Johansson, B.B. Cerebral lesions on magnetic resonance imaging, heart disease, vascular risk factors in subjects without stroke: a population-based study. Stroke 1994, 25, 929-934.

38. Ylikoski, A.; Erkinjuntti, T.; Raininko, R.; Sarna, S.; Sulkava, R.; Tilvis, R. White matter hyperintensities on MRI in the neurologically nondiseased elderly. Analysis of cohorts of consecutive subjects aged 55 to 85 years living at home. Stroke 1995, 26, 1171-1177.

39. Longstreth, W.T., Jr.; Manolio, T.A.; Arnold, A.; Burke, G.L.; Bryan, N.; Jungreis ,C.A.; Enright, P.L.; OLeary, D.; Fried, L. Clinical correlates of white matter findings on cranial magnetic resonance imaging of 3301 elderly people: the cardiovascular health study. Stroke 1996, 27, 1274-1282.

40. Hoshi, T.; Kitagawa, K.; Yamagami, H.; Furukado, S.; Hougaku, H.; Hori, M. Relations of serum high-sensitivity C-reactive protein and interleukin-6 levels with silent brain infarction. Stroke 2005, 36, 768-772.

41. Baker, D.L.; Desiderio, D.M.; Miller, D.D.; Tolley, B.; Tigyi, G.J. Direct quantitative analysis of lysophosphatidic acid molecular species by stable isotope dilution electrospray ionization liquid chromatography-mass spectrometry. Anal. Biochem. 2001, 292, 287-295.

42. Li, Z.G.; Yu, Z.C.; Wang, D.Z.; Ju, W.P.; Zhan, X.; Wu, Q.Z.; Wu, X.J.; Cong, H.M.; Man, H.H. Influence of acetylsalicylate on plasma lysophosphatidic acid level in patients with ischemic cerebral vascular diseases. Neurol. Res. 2008, 30, 366-369.

(C) 2010 by the authors; licensee MDPI, Basel, Switzerland. This article is an open access article distributed under the terms and conditions of the Creative Commons Attribution license (http://creativecommons.org/licenses/by/3.0/). 